\title{
Remote Learning in Psychiatry Residency Programs During COVID-19: Emergency Measure or Path for the Future?
}

\author{
Hager Koraym ${ }^{1} \cdot$ Stacey Kaltman ${ }^{1}\left[\right.$ Mihriye $^{\text {Mete }}{ }^{1,2} \cdot$ Mayada Akil $^{1,3}$
}

Received: 30 August 2021 / Accepted: 24 September 2021 /Published online: 6 October 2021

(c) Academic Psychiatry 2021

\section{To the Editor:}

The COVID-19 pandemic led to an unparalleled shift to remote didactics teaching within psychiatry residency programs. Though the Accreditation Council for Graduate Medical Education (ACGME) requires that psychiatry residencies protect didactics time [1], the format of the teaching is determined by individual programs. In-person didactics have traditionally been a part of the residency training experience, providing an opportunity to establish cohesiveness among residents and to get to know faculty. However, COVID-19 social distancing protocols forced many residency programs to rapidly shift to online learning.

Remote teaching triggered by a crisis is different from intentional online education. The latter is based on careful instructional design and employs a systematic approach to curriculum development and delivery. Typical planning and development takes 6-9 months [2]. Online curricula improve by the second or third iteration and are based on years of didactic theory and standards [2]. Intentional online education contrasts with what residency program directors recently were required to do: develop or adapt an online didactic curriculum, essentially overnight. Due to time limitations, many programs simply delivered their in-person curriculum remotely, thus contending with a number of obstacles, including content that does not lend itself to online delivery and faculty members who were not adequately prepared for this new teaching modality. Little is known about the impact of this change.

Stacey Kaltman

sk279@georgetown.edu

1 Georgtown University School of Medicine, Washington, DC, USA

2 MedStar Health Research Institute, Hyattsville, MD, USA

3 MedStar Georgetown University Hospital, Washington, DC, USA
We set out to evaluate the shift to online learning in psychiatry residency programs during the COVID-19 pandemic. We were interested in understanding the landscape pre-pandemic, how it changed with online teaching, and how these changes were received by program directors.

We conducted a cross-sectional online survey between July and November 2020. All psychiatry program directors $(n=245)$ listed on Fellowship and Residency Electronic Interactive Database Access (FREIDA) in 2020 were invited to participate. The survey contained multiple choice and open-ended questions. Descriptive statistical analysis was performed. Responses to open-ended questions were subjected to thematic analysis using principles of Consensual Qualitative Research [3].

Of the 245 psychiatry program directors contacted, 54 (22\%) completed the survey. Prior to the pandemic, $82 \%$ $(n=44)$ of responders reported their didactics were entirely in-person, $11 \%(n=6)$ reported in-person programming with some online teaching, and 7\% $(n=4)$ reported in-person programming with over half of the curriculum online. Post-pandemic, 98\% $(n=53)$ of responders reported using real-time online didactics. Thirteen percent $(n=7)$ of programs utilized online content from another organization/ institution; 33\% $(n=18)$ utilized self-directed study and 17\% $(n=9)$ utilized online pre-recorded lectures supplementary to real-time online lectures. The most frequently identified difficulties in adopting remote teaching were unfamiliarity with technology $(72 \% ; n=39)$ and discomfort with teaching learners virtually $(69 \% ; n=37)$. Zoom was the most common platform $(89 \% ; n=48)$, followed by WebEx $(37 \%$; $n=20)$, Skype (15\%; $n=8)$, Microsoft Teams $(9 \% ; n=5)$, BlueJeans $(7 \% ; n=4)$, and Google $(7 \% ; n=4)$. The majority of responders $(83 \% ; n=45)$ indicated their intention to modify pre-pandemic didactics once safe to reconvene in-person.

When given the opportunity to comment, program directors described numerous benefits to online learning, including ease and convenience in time and location, better attendance, and the ability to secure speakers who may not have 
been able to attend in-person due to logistical and financial hurdles. Program directors described aspects of technology that enriched the didactic experience such as the ability to record sessions and ask questions via the chat function, especially for residents who may be reluctant to speak up in groups.

Program directors also had a number of concerns. They consistently cited decreased attention, resident multitasking during lectures, and the less interactive and/or "less fun" nature of sessions. Not all didactic content and teaching strategies translated well to online learning. The technology itself also presented challenges, including faculty resistance, inconsistent internet connectivity, and screen fatigue. Program directors reported missing the interpersonal interactions that took place among co-residents and with faculty during in-person learning.

Since many learners and educators have now been vaccinated, restrictions imposed by the Centers for Disease Control and Prevention, hospital systems, and academic institutions are shifting. Program directors are now faced with the question of how to move forward with resident didactics. Our study is limited by the small sample size and low response rate. However, our findings suggest that a hybrid model would likely be favored by program directors. It may be difficult to return to the in-person-only didactics model after such a major and relatively successful shift to online learning. Online learning in non-emergent settings is not new. Reviews of the benefits of online learning versus faceto-face instruction demonstrate wide variability [4]. Clearly, not all online learning is created equal. Content is not the sole factor that affects learning. Adult learning principles [5] can and should be applied in either setting. The educator has to actively manage learning sessions, whether in-person or online, using interactive learning relevant to the learner. Small group discussions and interactive exercises can promote engagement, as can breaking up content into shorter digestible sections and assessing learning after each section. This emergency-imposed sudden shift to online didactics may be an opportunity for programs to review pedagogical approaches, reduce the use of in-person lectures, and move in the direction of interactive learning. Such changes will require that resources are made available to residency training programs, especially pertaining to curriculum design and faculty development.

\section{Declarations}

Ethics Approval All study procedures were approved by the Georgetown University IRB.

Disclosures On behalf of all authors, the corresponding author states that there is no conflict of interest.

\section{References}

1. Accreditation Council for Graduate Medical Education. ACGME program requirements for graduate medical education in psychiatry research. Chicago, IL: ACGME; 2020.

2. Hodges C, Moore S, Lockee B, Trust T, Bond AER. The difference between emergency remote teaching and online learning. EDUCAUSE Review. 2020.

3. Hill CE, Knox S. Essentials of consensual qualitative research. Washington, DC: American Psychological Association; 2021.

4. Cook DA, Levinson AJ, Garside S, Dupras DM, Erwin PJ, Montori VM. Internet-based learning in the health professions: a metaanalysis. JAMA. 2008;300(20):1181-96.

5. Knowles M. Andragogy in action. San Francisco: Jossey-Bass; 1984.

Publisher's Note Springer Nature remains neutral with regard to jurisdictional claims in published maps and institutional affiliations. 